\title{
New Scale-up Technologies for Hydrogenation Reactions in Multipurpose Pharmaceutical Production Plants
}

\author{
Thierry Furrera, Benedikt Müller ${ }^{a}$, Christoph Hasler ${ }^{a}$, Bernhard Berger ${ }^{b}$, Michael K. Levis ${ }^{b}$, and \\ Andreas Zogg ${ }^{\star a}$
}

\begin{abstract}
The classical scale-up approach for hydrogenation reaction processes usually includes numerous laboratory- and pilot-scale experiments. With a novel scale-up strategy, a significant number of these experiments may be replaced by modern computational simulations in combination with scale-down experiments. With only a few laboratory-scale experiments and information about the production-scale reactor, a chemical process model is developed. This computational model can be used to simulate the production-scale process with a range of different process parameters. Those simulations are then validated by only a few experiments in an advanced scale-down reactor. The scale-down reactor has to be geometrically identical to the corresponding production-scale reactor and should show a similar mass transfer behaviour. Closest similarity in terms of heat transfer behaviour is ensured by a sophisticated 3D-printed heating/cooling finger, offering the same heat exchange area per volume and overall heat-transfer coefficient as in production-scale. The proposed scale-up strategy and the custom-designed scale-down reactor will be tested by proof of concept with model reactions. Those results will be described in a future publication. This project is an excellent example of a collaboration between academia and industry, which was funded by the Aargau Research Fund. The interest of academia is to study and understand all physical and chemical processes involved, whereas industry is interested in generating a robust and simple to use tool to improve scale-up and make reliable predictions.
\end{abstract}

Keywords: Agitated vessel · Hydrogenation · Process modelling · Quality by Design (QbD) · Scale-up / Scale-down

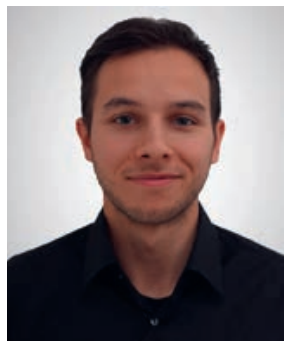

Thierry Furrer completed his apprenticeship as a chemical laboratory technician at F. Hoffmann-La Roche AG in 2015. In 2020 he obtained his BSc in Molecular Life Sciences with specialisation in chemistry from the University of Applied Sciences and Arts Northwestern Switzerland (FHNW). During his master studies he is now working for the research group of Prof. Dr. Andreas Zogg at the FHNW HLS. His main focus is the development of a novel scale-down reactor and the improvement of computational chemical process models for scale-up purposes.

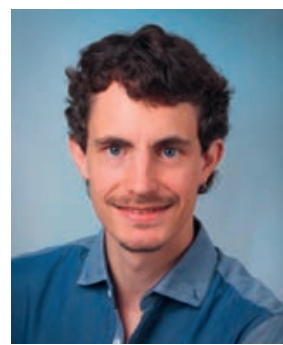

Benedikt Müller completed his apprenticeship as chemical operator at F. HoffmannLa Roche AG in 2002. He practiced his profession at the same company (since 2003 DSM Nutritional Products) in Sisseln (AG), where he was part of the start-up team of the vitamin E production. While working as laboratory technician for Willy A. Bachofen AG, in 2011 he obtained a Swiss Maturity Diploma. In 2018 he obtained his MSc in Industrial Life Sciences (major: Molecular Technologies)

*Correspondence: Prof. A. Zogg ${ }^{\text {a }}$ E-mail: andreas.zogg@fhnw.ch

anstitute for Chemistry and Bioanalytics, University of Applied Sciences and Arts Northwestern Switzerland, School of Life Sciences (FHNW HLS),

Hofackerstrasse 30, CH-4132 Muttenz

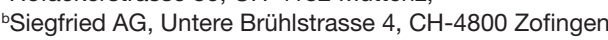

from the University of Applied Sciences and Arts Northwestern Switzerland (FHNW). After that he was part of the research group of Prof. Dr. Andreas Zogg and was responsible for establishing the laboratory of process technology.

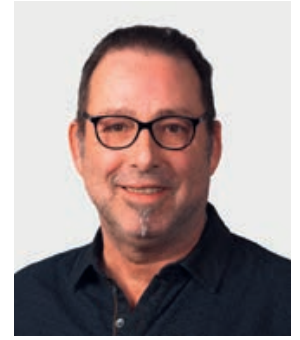

Christoph Hasler completed his apprenticeship as a chemical technician at CibaGeigy AG (later Ciba SC) in 1991. He practiced his profession at the same company until 2005. From 1998 he held a shift supervisor position in pilot-scale and in 2006 obtained his advanced federal diploma of higher education as a certified chemical technician (HFP). From 2006 to 2010 he worked as shift supervisor for Valorec Services AG. He then moved internally to take up a position as head of engineering (production) and education (chemical technicians). In 2017 he joined the FHNW as a technician specialist and since 2020 he is part of the research group of Prof. Dr. Andreas Zogg.

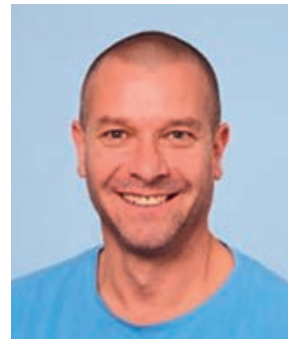

Bernhard Berger completed his apprenticeship as a lab technician specialised in chemical synthesis at Sandoz Research Institute Bern in 1991. He practiced his profession at the same company (from 1996, Novartis) in Bern and Basel until 2000. In 2000 he moved to the Novartis Health \& Functional Food division in Neuenegg and worked in the analytical department. In 2003 he joined Siegfried AG and started working in the chemical development $(\mathrm{R} \& \mathrm{D})$ department in 
Zofingen. In 2006 he obtained his advanced federal diploma of higher education as a senior scientific laboratory technician (HFP). He has since taken over the responsibility for the RC1 measurements (reaction calorimetry) as part of thermal process safety. Additionally, his work is focused on process modelling to ensure safe scale-up of chemical processes regarding safety and quality.

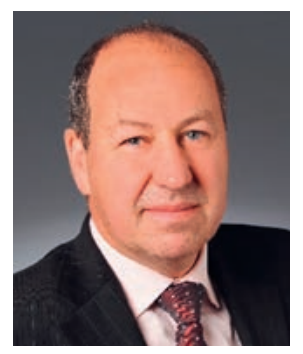

Dr. rer. nat. Michael Karl Levis obtained his $\mathrm{PhD}$ in organic chemistry from the Albert Ludwigs University in Freiburg, Germany under the guidance of Prof. Dr. Schmittel and Prof. Dr. Rüchardt in 1994. In 1995 he joined Siegfried AG and headed positions in analytical development, chemical development and process optimization including a 3 -year internship at the Siegfried site in Pennville, USA. He is the Head of Process Technologies (R\&D) and is principal scientist for Particle Technologies within the Siegfried group.

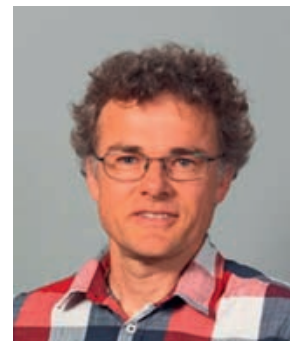

Prof. Dr. Andreas Zogg obtained his diploma in technical chemistry (1999) and a $\mathrm{PhD}$ in technical science (2003) from the ETH Zurich. After a post-doctoral stay at Ciba SC, he became project leader within the process development department of Ciba SC (2004), polymer additives. In 2009 he joined F. Hoffmann-La Roche AG as a senior scientist in pharma technical development and was responsible for the development of new technologies as well as technically demanding chemical processes. In 2017 he joined Novartis Pharma AG as safety lab expert in pharma chemical and analytical development. Since 2018 he is professor for physical chemistry, process modelling and reaction technology at the University of Applied Sciences and Arts (FHNW). His research focuses on the development of new reaction technologies and process modelling approaches for the safe and direct scale-up of chemical processes. Since 2019 he is president and cofounder of the association 'Miniplant 4.0'.

\section{Introduction}

The main goal of a chemical process scale-up is usually defined as the reproduction of laboratory-scale results on a larger scale. Same conversions, yields, selectivity and preferably even improvements of those parameters should be achieved. ${ }^{[1]}$ The classical scale-up strategy is based on numerous laboratory-scale experiments and often includes an intermediate batch-scale, a so-called pilot-scale campaign, to approach production-scale. Nevertheless, it is possible that a well-defined process delivers good results in laboratory- and pilot-scale, and then fails to achieve the required quality or performance in a production-scale batch. ${ }^{[2]}$ Such undesired deviations occur especially if mixing influences the process selectivity and thus some of the reactions involved are both fast and mass transport dependent. ${ }^{[3,4]}$ For a more efficient and controlled process scale-up, it is possible to simulate the behaviour of the production-scale reaction process based on a dynamic process model of the production-scale reactor. These models are usually based on reaction kinetics, reaction enthalpies and dynamic physical models for heat and mass transfer of production-scale reactors. ${ }^{[5-7]}$ With information and knowledge gained from the development of a chemical process model the establishment of the design space can be strongly supported. ${ }^{[8,9]}$ Product and process understanding is a key element of Quality by Design (QbD). Quality cannot solely be tested in products and should therefore be built into the product by design. ${ }^{[10]}$ Efforts to make scale and process parameter changes predictable and reliable are therefore highly appreciated by regulatory authorities.

To validate the chemical process model, the reaction can be carried out in a scaled-down version of the production-scale reactor. ${ }^{[11]}$ In this validation experiment, not only closest similarity in mass and heat transfer but also in geometry and hydrodynamics must be maintained. ${ }^{[12,13]}$

Zufferey ${ }^{[14]}$ used the $\mathrm{RC} 1$ reaction calorimeter commercialised by Mettler Toledo ( $2 \mathrm{~L}$ ) to predict and experimentally imitate the heat transfer behaviour of production-scale reactors $(40 \mathrm{~L}$ to $25 \mathrm{~m}^{3}$ ). The prediction is based on two online heat balances. One determines the actual reaction heat measured by the calorimeter with known heat transfer behaviour. The other numerically simulates the theoretical reaction mixture temperature of the production-scale reactors, based on previously generated and validated dynamic heat transfer models of the same production-scale reactors. However, with this approach, the heat transfer area to volume ratio and therefore also the inner wall temperature of the laboratory-scale calorimeter deviate significantly from production-scale reactors.

In another approach, suggested by Kupr and Hub, ${ }^{[15]}$ the heat transfer area of the laboratory-scale calorimeter was modified to have the same heat transfer area to volume ratio as production-scale reactors. This was achieved by lowering the heat transfer fluid level in the glass jacket of the calorimeter. Furthermore, the magnitude of the heat transfer coefficient was also kept equal to that of the production reactors by changing the flow rate of the heat transfer fluid. With this approach, the authors were able to imitate the heat transfer behaviour of stainless steel and enamelled reactors from $160 \mathrm{~L}$ to $16000 \mathrm{~L}$. Ashe ${ }^{[16]}$ proposed a similar approach with different intentions. He developed a continuous stirred tank reactor with a variable number of small jacket elements. He suggested that the measurement of exchanged heat in production-scale reactors with such jackets is improved. Furthermore, the working area of the jacket can be limited to liquid-covered surfaces. This should avoid thermal damage to heat-sensitive products.

The approaches mentioned did not investigate the scale dependency of the mixing and mass transfer performance and neglected geometrical similarity to some extent.

Siegfried AG, a well-known CDMO for the pharmaceutical industry, and the University of Applied Sciences and Arts Northwestern Switzerland (FHNW) are together developing a scale-up strategy based on the current state of the art. This strategy aims to achieve a safer, more direct and controlled introduction of hydrogenation reaction processes into production-scale. Hydrogenation reactions are very demanding in terms of process safety and chemistry. In Fig. 1 parameters influencing hydrogenation processes are illustrated in blue. With the proposed scale-up strategy, adequate process performance (green) should be achieved with only a few laboratory-scale experiments. This strategy could have a wide field of application since many complex multi-step syntheses have at least one hydrogenation step.

\section{Scale-up Strategy}

The guideline for this article will be a flowchart illustrating the proposed scale-up strategy (Fig. 2). The centrepieces of this scale-up strategy are a scale-down of the production-scale reactor and a computational dynamic process model. For the design of both, specific laboratory- and production-scale data are required. With the dynamic model, the parameters of the production-scale reaction process can be adjusted to achieve the desired product quality and production time. The simulated process with the optimized process parameters is then verified in an advanced, customized scale-down experiment. If the results of this experiment agree with the simulation, the process may be scaled-up directly to production-scale. The production-scale process then generates valuable dynamic data. On one hand, those data can be used for 


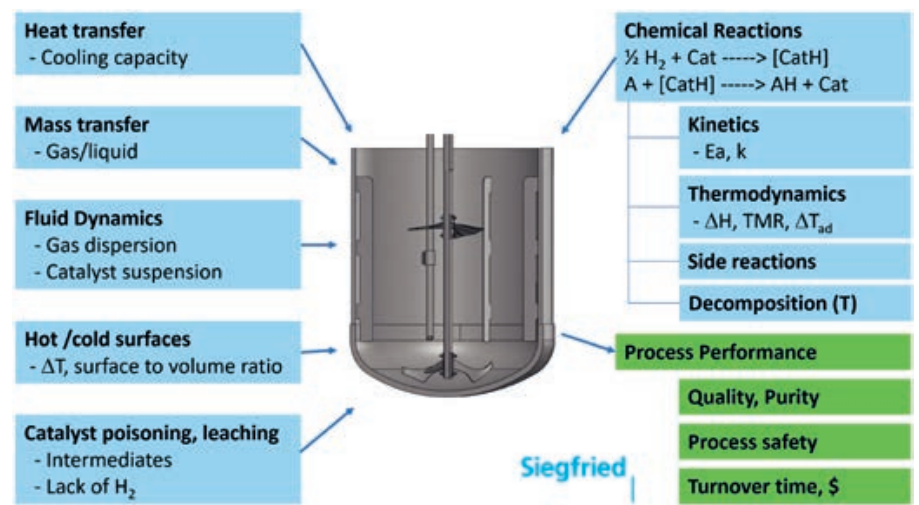

Fig. 1. CAD drawing of agitated production-scale autoclave (middle), main parameters influencing a hydrogenation process (blue) and process performance indicators (green).

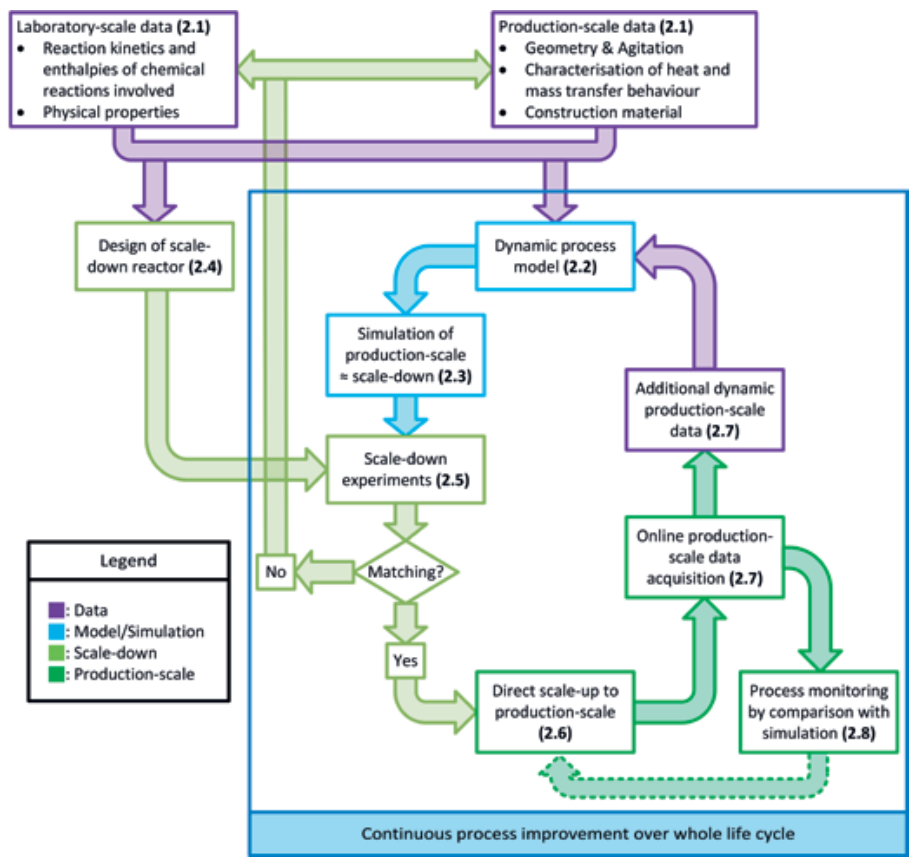

Fig. 2. Flowchart illustrating the proposed scale-up strategy (numbers in bold indicate the chapter numbers which refer to this article).

online process monitoring and on the other, to continuously improve the dynamic process model.

\subsection{Laboratory and Production-Scale Data}

Laboratory-scale data include reaction kinetics, reaction enthalpy and the physical properties of the reaction mixture. The required production-scale data include geometry, agitation, construction material and a characterization of the dynamic behaviour of the production-scale reactor.

\subsubsection{Reaction Kinetics and Enthalpy}

The description of the chemical process is an important part of a dynamic process model. If possible, the kinetic parameters (reaction orders, activation energies, rate constants) and reaction enthalpy of all reactions occurring in the process should be known. With a stirred-tank reaction calorimeter ${ }^{[17]}$ and an appropriate analytical method, those parameters can be determined with only a few experiments. An interesting approach to generate these preliminary data by online calorimetric and non-calibrated infrared data was investigated by Zogg et al. ${ }^{[18]}$ They used such data to identify chemical parameters of acetic anhydride hydrolysis with a combined evaluation algorithm. For an accurate characterization of more complex multi-step reactions, it is suggested to use further process analytical technologies (PAT) to follow the substrate, product, intermediates and by-products concentrations.

For gas-liquid reactions such as hydrogenations, the mass transfer of gaseous reactants into the liquid phase must be considered. Therefore, mass transfer correlations are generally determined experimentally and included in a computational, dynamic model of the reaction calorimeter. With subsequent experiments in this reaction calorimeter, the kinetic and thermodynamic parameters are identified by simulating the experimental system and minimizing the error to the measured data. This approach is described in literature with different software and optimization tools. ${ }^{[5,19]}$

At Siegfried AG the $\mathrm{RC} 1$ reaction calorimeter combined with an automatic sampling system, offline analytics and spectroscopic PAT tools are used to generate experimental data. The kinetic and thermodynamic parameters of the corresponding reactions and involved side reactions are then determined with Dynochem ${ }^{\circledR} .{ }^{[20]}$ Models including up to 22 competing reactions were developed to describe the reaction process in a precise and robust computational model.

\subsubsection{Physical Properties of the Reaction Mixture}

Heat and mass transfer behaviour are strongly linked to the physical properties (density, heat capacity, thermal conductivity and viscosity) of the reaction mixture and therefore also to their temperature dependency. Thus, those properties are determined experimentally if they are not already known. Averaging the properties of the start and end reaction mass improves the results. Only considering the known physical properties of the solvent and neglecting the influence of the other components represents a first approximation if no further measurements are possible.

\subsubsection{Geometry, Agitation and Construction Material of the Production-Scale Reactor}

For the design of the scale-down reactor and estimation of the production-scale heat and mass transfer correlations, specific information about the production-scale reactor is needed. This includes, among other things, specific geometric information (bottom, jacket, stirrer and cylindrical part) and the construction material. Furthermore, the physical properties of the construction material (density, specific heat capacity and thermal conductivity), the mass flow and temperature-dependent physical properties of the heat transfer fluid (density, heat capacity, thermal conductivity and viscosity) must be known. If the mass flow of the heat transfer fluid is not measured, it is determined or estimated in relation to the inlet temperature of the heat transfer fluid.

\subsubsection{Characterisation of Production-Scale Reactor}

To validate and adjust the heat and mass transfer part of the dynamic process model, experimental data of the production-scale reactor are used, i.e. isothermal and ramped heating/cooling experiments and gas (hydrogen) uptake experiments. Those experiments are carried out with varying jacket temperatures, fill levels, solvents and agitation speeds.

\subsection{Dynamic Process Model}

A computational process model to simulate the reaction process in the production-scale reactor is developed for each new chemical process. The model numerically simulates the concentrations of all components in the reaction mixture and gas phase, as well as the temperatures of all simulated sections (reaction mixture, gas phase, reactor wall and heat transfer fluid (jacket)).

The model can be subdivided into three parts (chemical reactions, heat transfer and mass transfer). It is assumed that the gas phase has the same temperature as the reaction mixture. Therefore, it is not considered in the heat transfer part. 
In this article only the development of the heat transfer part will be described in detail. A description of the remaining parts is planned in a future publication. The model was developed using MATLAB ${ }^{[21]}$ and implemented into Dynochem. ${ }^{[20]}$ If a process model of the same production-scale reactor is already available, only the part specifying the chemical reaction needs to be adjusted within Dynochem. The input needed and output generated by the finalized dynamic Dynochem process model is illustrated in Fig. 3.

\section{Model input \\ - Process parameters: \\ - Jacket inlet temperature (dynamic, $T_{\text {inlet }}$ ) \\ - Agitation speed (dynamic) \\ - Gas phase pressure (dynamic) \\ - Initial amount of substance of all components \\ - Initial temperature of reaction mixture \\ - Physical properties of the reaction mixture \\ - Kinetic and thermodynamic parameters describing the chemical reactions \\ - Heat and mass transfer correlations}

\section{Model output}

- Amount of substance of all components (dynamic)

- $\quad$ Reaction mixture temperature (dynamic, $T_{R}$ )

- Jacket outlet temperature (dynamic, $T_{J}$ )

- Wall temperature (dynamic, $T_{W}$ )

Fig. 3. Input and output of the dynamic process model.

\subsubsection{Chemical Reactions}

This part of the model computes the rates of all reactions at each time step according to the Arrhenius equation. It uses kinetic parameters determined by the method described in section 2.1.1. With the reaction rate it is possible to compute, for each time step, the concentration change of each component and, by considering the reaction enthalpies, the heat flow due to the reactions involved.

\subsubsection{Heat Transfer}

For the heat transfer part, the reactor is divided into three different sections. These sections are illustrated in Fig. 4: 1) Heat transfer fluid, 2) Reactor wall, 3) Reaction mixture. The temperature within a section is assumed to be homogeneous. The temperature of each section is computed by numerical integration of Eqn. (1), where all variables except time are section specific:

$$
\frac{d T}{d t}=\frac{\dot{Q}_{a c c u}}{m \cdot c p}
$$

The wall of the stainless-steel hydrogenation autoclave, between the reaction mixture and the jacket, is relatively thick and heavy. Therefore, it was important to include the influence of the reactor wall on the dynamic heat transfer behaviour of the reactor. In the developed model, the temperature of the wall $\left(T_{W}\right)$ was simulated at half its thickness.

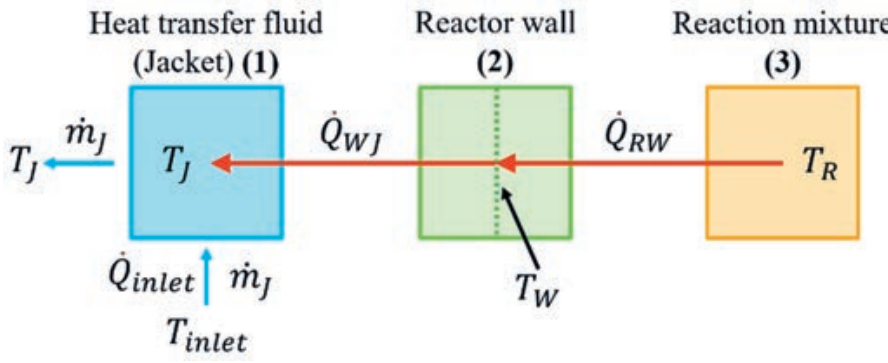

Fig. 4. Structure of heat transfer model of production-scale reactor.

With a heat balance for each section, the heat flows due to accumulation $\left(\dot{Q}_{\text {accu }}\right)$ are computed. The heat flow due to accumulation in the reaction mixture $\left(\dot{Q}_{a c c u, R}\right)$ is computed according to Eqn. (2):

$$
\dot{Q}_{a c c u, R}=\dot{Q}_{\text {react }}-\dot{Q}_{R W}
$$

The heat flow from the reaction mixture to the reactor wall $\left(\dot{Q}_{R W}\right)$ is computed according to Eqn. (3):

$$
\dot{Q}_{R W}=U_{R W} \cdot A_{R W} \cdot\left(T_{R}-T_{W}\right)
$$

With the geometry of the reactor, the density and mass of the reaction mixture, the heat transfer area $\left(A_{R W}\right)$ is computed dynamically. The heat transfer coefficient $\left(U_{R W}\right)$ is determined as stated in Eqn. (4):

$$
U_{R W}=\left(\frac{d_{V}}{N u_{R} \cdot \lambda_{R}}+\frac{\text { thickness }_{W}}{2 \cdot \lambda_{W}}\right)^{-1}
$$

The right part of this definition includes the thermal resistance of half the wall thickness $\left(\right.$ thickness $_{W}$ ). The left side defines the heat transfer from the inner surface of the stirred reactor to the surface of the wall due to forced convection. ${ }^{[22]}$ The Nusselt number $\left(N u_{R}\right)$ is computed according to Eqn. (5):[22]

$$
N u_{R}=C_{1 \mid 2} \cdot R e_{R}^{\frac{2}{3}} \cdot \operatorname{Pr}_{R}^{\frac{1}{3}} \cdot\left(\frac{\eta_{R}}{\eta_{R, W}}\right)^{0.14}
$$

The agitator used has two agitator units as can be seen in Fig. 1. If only the first agitator unit is immersed in the reaction mixture, parameter $C_{1}$ is used. If both units are in contact with the reaction mixture, parameter $C_{2}$ is used. For the transitional area a weighted combination of both is used. Furthermore, the impeller Reynolds number $\left(R e_{R}\right)$ is defined by Eqn. (6):

$$
R e_{R}=\frac{n \cdot d_{A}^{2} \cdot \rho_{R}}{\eta_{R}}
$$

The heat flow due to accumulation in the reactor wall $\left(\dot{Q}_{a c c u, W}\right)$ is computed according to Eqn. (7):

$$
\dot{Q}_{a c c u, W}=\dot{Q}_{R W}-\dot{Q}_{W J}
$$


The computation of the heat flow between the reactor wall and the jacket $\left(\dot{Q}_{W J}\right)$ is similar to the computation of $\left(\dot{Q}_{R W}\right)$ and is defined by Eqn. (8):

$$
\dot{Q}_{W J}=U_{W J} \cdot A_{W J} \cdot\left(T_{W}-T_{J}\right)
$$

Since the path of the heat transfer fluid does not change, the heat transfer area $\left(A_{W J}\right)$ is set as constant and calculated with knowledge of the reactor geometry. The heat transfer coefficient $\left(U_{W J}\right)$ is computed according to Eqn. (9):

$$
U_{W J}=\left(\frac{4 \cdot d_{e}}{N u_{J} \cdot \lambda_{J}}+\frac{\text { thickness }_{W}}{2 \cdot \lambda_{W}}\right)^{-1}
$$

Further, the Nusselt number is defined by Eqn. (10):[23]

$$
N u_{J}=C_{3} \cdot R e_{J}^{C_{4}} \cdot \operatorname{Pr}_{J}^{C_{5}} \cdot\left(\frac{\eta_{J}}{\eta_{J, W}}\right)^{0.14} \cdot\left(1+3,5 \cdot \frac{4 \cdot d_{e}}{d_{c}}\right)
$$

Here, the Reynolds number is computed according to Eqn. (11):

$$
R e_{J}=\frac{4 \cdot d_{e} \cdot v_{J} \cdot \rho_{J}}{\eta_{J}}
$$

The heat flow due to accumulation in the jacket $\left(\dot{Q}_{a c c u, J}\right)$ is computed according to Eqn. (12):

$$
\dot{Q}_{a c c u, J}=\dot{Q}_{W J}+\dot{Q}_{\text {inlet }}
$$

As in the reaction mixture section, the jacket (heat transfer fluid) is assumed to have no spatial variation in temperature. Since the fluid which leaves the jacket has the same temperature as the section, there will be no heat flow out of the jacket. However, the temperature of the oil which enters the jacket $\left(T_{\text {inlet }}\right)$ can differ compared to the jacket temperature $\left(T_{j}\right)$. The heat flow due to this inflow is computed by Eqn. (13):

$$
\dot{Q}_{\text {inlet }}=\dot{m}_{J} \cdot c p_{J} \cdot\left(T_{\text {inlet }}-T_{J}\right)
$$

The parameters $C_{1}$ to $C_{5}$ and the relevant mass of the reactor wall $\left(m_{W}\right)$ were identified with a least squares optimization based on simulated and measured temperatures of different heating and cooling experiments. For this, the Surrogate Optimization Algorithm[24] implemented in the MATLAB ${ }^{[21]}$ Global Optimization Toolbox was applied. The boundaries for parameters $C_{1}$ to $C_{5}$ were set to $-1+15 \%$ of the expected literature values. ${ }^{[22,23]}$ The boundaries for the relevant reactor wall mass were set to $3000-9500 \mathrm{~kg}$. The value estimated from the geometry and density was $6250 \mathrm{~kg}$.

The simulation result of one of the heating and cooling experiments of the production-scale reactor can be seen in Fig. 5. Based on the jacket inlet temperature $\left(T_{\text {inlet }}\right)$ the process model is able to simulate the reaction mixture temperature $\left(T_{R}\right)$, the wall temperature $\left(T_{W}\right)$ as well as the jacket outlet temperature $\left(T_{J}\right)$. As can be seen in Fig. 5 the simulated temperatures $\left(T_{R}\right.$ and $\left.T_{J}\right)$ agree very well with the corresponding measured data.

\subsubsection{Mass Transfer}

For multiphase reactions, such as hydrogenations, the conversion rate can be controlled by the rate of interphase mass transfer. ${ }^{[3]}$ Therefore, it is also important to include the dynamics of the mass transfer (gas-liquid) in the process model.

If the transport is too slow, reaction kinetics will slow down, so that side reactions become prominent or dangerous intermediates may accumulate. If the catalyst depletes from hydrogen, catalyst poisoning or heavy metal, leaching is possible. Therefore, hydrogen mass transport is relevant for both process quality and safety.

The rate of mass transfer across the phase boundary can be described according to Eqn. (14):

$$
\frac{d c_{L}}{d t}=k_{L} a \cdot\left(c_{L, s a t}-c_{L}\right)
$$

Analogous to heat transfer, the mass transfer can also be divided into individual resistances. Diffusion in the gas phase is usually several powers of orders of magnitude faster than in the liquid phase. Therefore gas-phase transfer resistance can be neglected

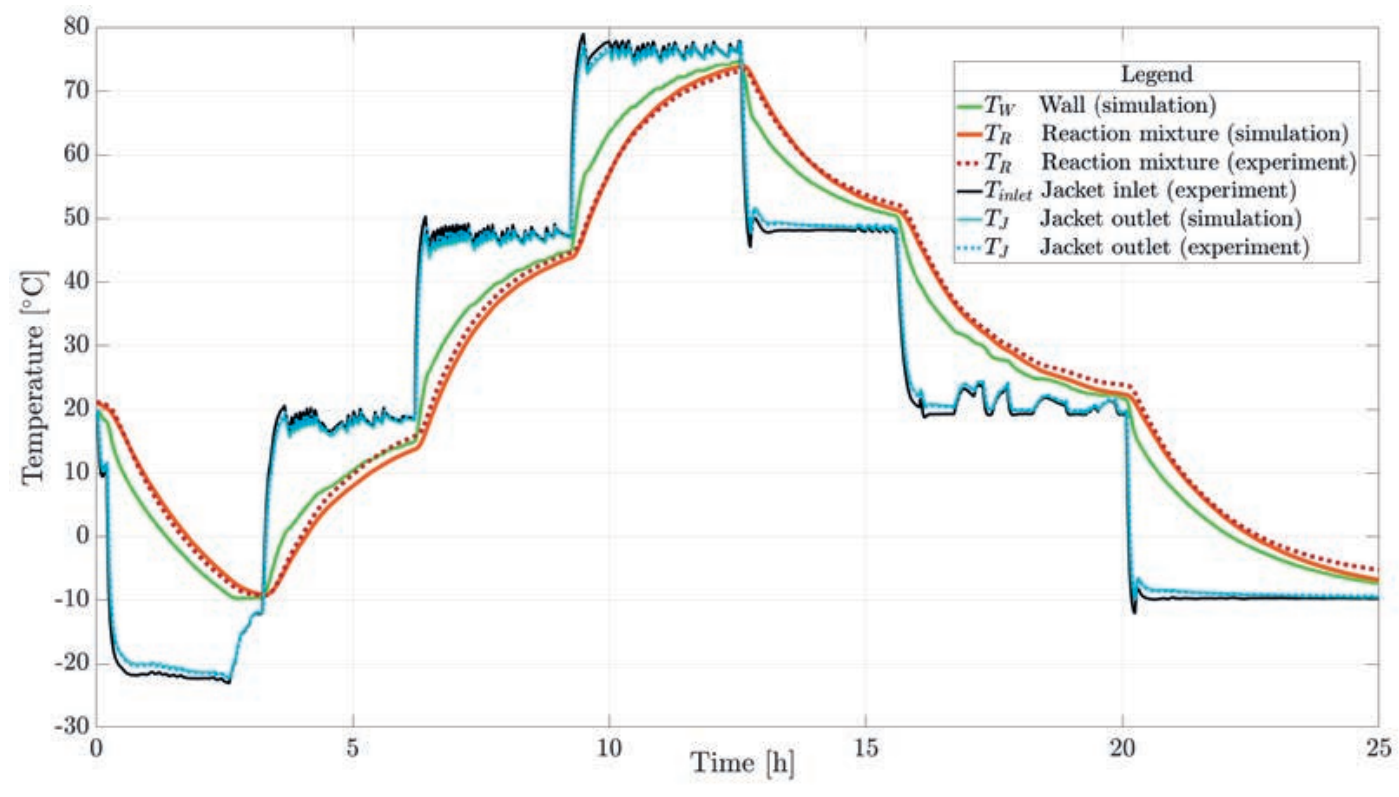

Fig. 5. Simulated heat transfer behaviour of the $4 \mathrm{~m}^{3}$ productionscale agitated autoclave filled with $3075 \mathrm{~kg}$ methanol (only $T_{\text {inlet }}$ imposed) 
and the liquid-phase transfer resistance $\left(k_{L}\right)$ equals to the overall and speed-determining transfer resistance. ${ }^{[12]}$ Since it would be a large effort to measure the phase interface, the product of the resistance $\left(k_{L}\right)$ and the interface area per volume $(a)$ is usually considered and measured as a unit $\left(k_{L} a\right){ }^{[12]}$ The saturation concentration $\left(c_{L, s a t}\right)$ is described by the solubility of the hydrogen in the reaction mixture. It is assumed to be equal to the solubility in the solvent only. The pressure dependency and absolute values of the saturation concentration can be described by Henry's law.[25]

At the current stage of the project, the $k_{L} a$ value for hydrogen in the production-scale reactor was experimentally determined for two different solvent systems at varying temperature, fill level and agitation speed. The dependency on those parameters was then incorporated into the dynamic process model. It is assumed that the mass transfer of the reaction mixture behaves as though the reaction mixture is purely one of the measured solvents.

The mass transfer part of the dynamic process model will be further investigated. The mass transfer will be incorporated into the model in the same way as the heat transfer part and hence the dependency of the physical properties of the reaction mixture on the gas-liquid mass transfer will be considered. The results will be described in a future publication.

\subsection{Simulation of Production-Scale ( $\approx$ Scale-down)}

With the complete dynamic process model, it is possible to simulate chemical reaction processes in production-scale reactors. The process parameters shown in Fig. 3 can be adjusted so that the simulation results meet the desired specifications. Since the scale-down experiment should imitate the production-scale reactor, the simulation will also predict the reaction process in the scale-down experiment. With the dynamic process model, it is also possible to identify the limiting factor for the reaction process (e.g. heat or mass transfer).

\subsection{Design of Scale-down Reactor}

The scale-down reactor should be able to validate the simulations of the production processes and it should therefore imitate the production-scale reactor as accurately as possible. For this, its geometry, heat transfer behaviour and mass-transfer behaviour (agitation and flow profile) must be similar. Siegfried AG and the University of Applied Sciences and Arts Northwestern Switzerland are currently developing and building a scale-down reactor of a $4 \mathrm{~m}^{3}$ hydrogenation autoclave (Fig. 1) in cooperation with Premex Solutions GmbH. This reactor will be installed in the new Process Technology Center (PTC) of the FHNW HLS.

\subsubsection{Geometric Similarity}

The vessel itself and the agitator will be made of glass having the same geometry as the production-scale autoclave. With an inner diameter of about $120 \mathrm{~mm}(\sim 1 \mathrm{~L})$, the scale-down reactor is significantly smaller than its original with an inner diameter of $1720 \mathrm{~mm}$ ( 4000 L). The gas injection tube and baffles are also designed to show good geometrical similarity. Those will be made from stainless-steel, as in the production-scale reactor. One of the four baffles will be replaced by an automated sampling system and another by a heating/cooling finger (H/C-finger, Fig. 6) which is geometrically identical to the baffle.

\subsubsection{Laboratory Safety}

The glass vessel will be kept in a bigger $(100 \mathrm{~L})$ pressure resistant (10 bar) stainless-steel reactor which will function as safety and isolation container (Fig. 7). This stainless-steel container prevents a severe laboratory accident in case the glass autoclave bursts. Possible reasons for bursting could be an unexpected runaway, pressure build up or material failure. During normal operation the stainless-steel container takes on the function of a blow down tank (see red line in Fig. 8). The scale-down reactor is de-

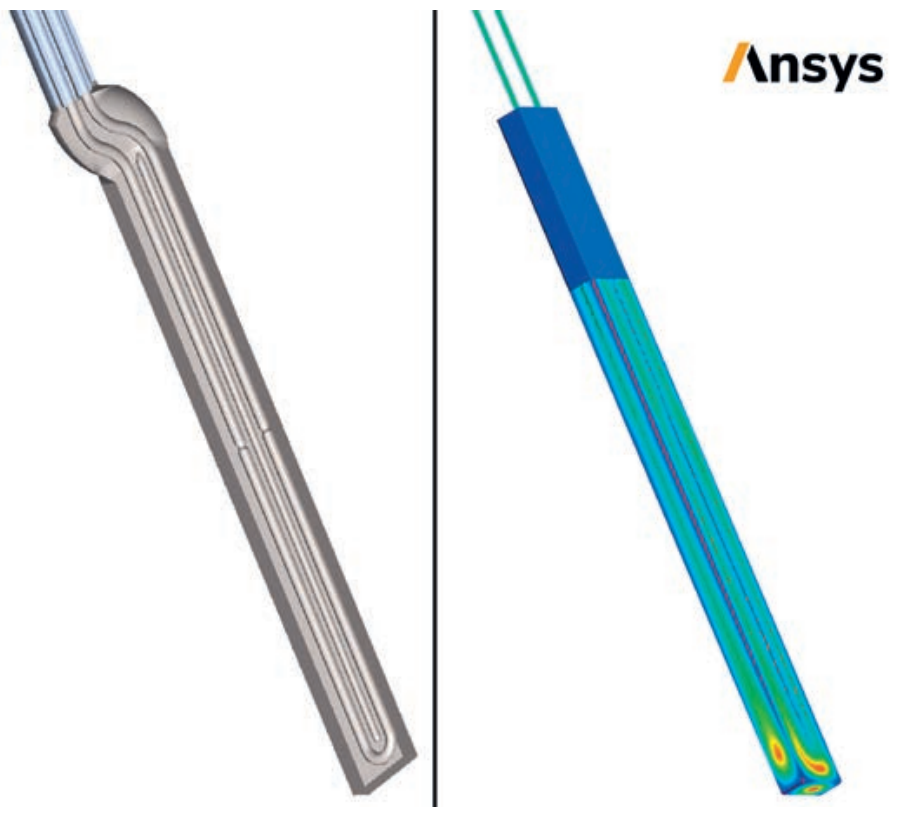

Fig. 6. Cross section view of final H/C-finger design (left). Visualization of ANSYS ${ }^{\circledR}$ Fluent ${ }^{\circledR}$ CFD results (right).

signed so that it is possible to mount a bigger glass reactor $(5 \mathrm{~L})$ without major changes. It will be possible to equip this bigger glass reactor with a pneumatic bottom valve to allow an emergency quench of the reaction mixture with a suitable solution in the 100 L container.

\subsubsection{Similarity of Heat Transfer Behaviour}

Unlike ordinary laboratory pressure autoclaves this reactor will not be jacketed. The isolating function of the $100 \mathrm{~L}$ container will minimize heat exchange between the glass wall and its environment (quasi adiabatic behaviour). For this, the temperature of the safety container will be kept at the same temperature as the reaction mixture in the glass vessel.

The heat transfer with the reaction mixture will be realized using an H/C-finger (Fig. 6) which imitates the heattransfer behaviour of the production-scale reactor. Silicon oil as heattransfer fluid will flow through the H/C-finger. The control of the silicon oil inlet temperature is realized by adjusting the mixing ratio of the oil at two different temperatures. With this approach it is possible to mimic fast inlet temperature changes which are common in production-scale reactors.

The H/C-finger is designed to meet the following conditions:

- similar geometry to a baffle of the production-scale reactor;

- similar volume to heat transfer area ratio as the production-scale reactor;

- similar overall heat transfer coefficient as the production-scale reactor;

- similar construction material as the production-scale reactor wall.

If these conditions are fulfilled, the heat transfer behaviour of the scale-down reactor will be very close to that of its corresponding production-scale reactor. Furthermore, the surface temperature of the heat exchangers (production-scale wall and $\mathrm{H} / \mathrm{C}$ finger) will be similar. Therefore, it is possible to imitate potential chemical reactions close to or on hot/cold surfaces. As illustrated in Fig. 1, hot/cold surfaces are an important process parameter for hydrogenation reactions.

The H/C-finger was designed using computational fluid dynamics (CFD). The results of these computations were then combined with the dynamic process model. ANSYS ${ }^{\circledR}$ Fluent ${ }^{\circledR[26}$ was used for the CFD calculations. The heat flow from the oil to 
the solid part of the finger was simulated for various oil temperatures and the resulting heat transfer coefficient (Fig. 6, right) was compared to that observed in the production-scale reactor heating/cooling experiments. The geometry of the channel in the $\mathrm{H} / \mathrm{C}$-finger was then modified until the computed heat transfer coefficient matched the one obtained from the production-scale process model.

\subsubsection{Similarity of Mass Transfer Behaviour}

The geometrical similarity of the scale-down and production-scale reactor should contribute to the similarity of mass transfer. The agitation speed of the scale-down reactor is used to adjust the mass transfer. With gas (hydrogen) uptake experiments at varying temperatures, agitator speeds and fill levels, a mass transfer correlation will be derived for the scale-down reactor. The agitator speed can then be set so that the mass transfer is equal for both scales. A similar approach was proposed by Benz. ${ }^{[27]}$ However, for heterogenic reactions, dispersion needs to be considered; the agitation speed should be fast enough to disperse the mixture.

\subsubsection{Final Design}

The final scale-down reactor design is illustrated by the three-dimensional drawing (Fig. 7) and the schematic representation (Fig. 8). The inside temperature of the bigger stainless-steel container and lid can be controlled by an external thermostat, using tempered silicon oil flowing through a channel in the lid and copper tubes in the isolation container. The surfaces in frequent contact with the reaction mixture will be made from highly corrosion resistant Hastelloy C-22.

\subsection{Scale-down Experiments}

With this custom-designed scale-down reactor, experiments of the simulated reaction processes can be performed and the simulation can be validated. This experiment must be carried out under exactly the same conditions as simulated and planned in the production-scale reactor. If the experimental results agree with the simulation, it can be assumed that the reaction process will lead to the same results in production-scale. If not, however, the reasons for the deviation need to be investigated. It might be possible that laboratory- or production-scale data need to be extended or measured again.

In this phase of development, PAR (Proven Acceptable Range) experiments are usually performed to demonstrate the robustness

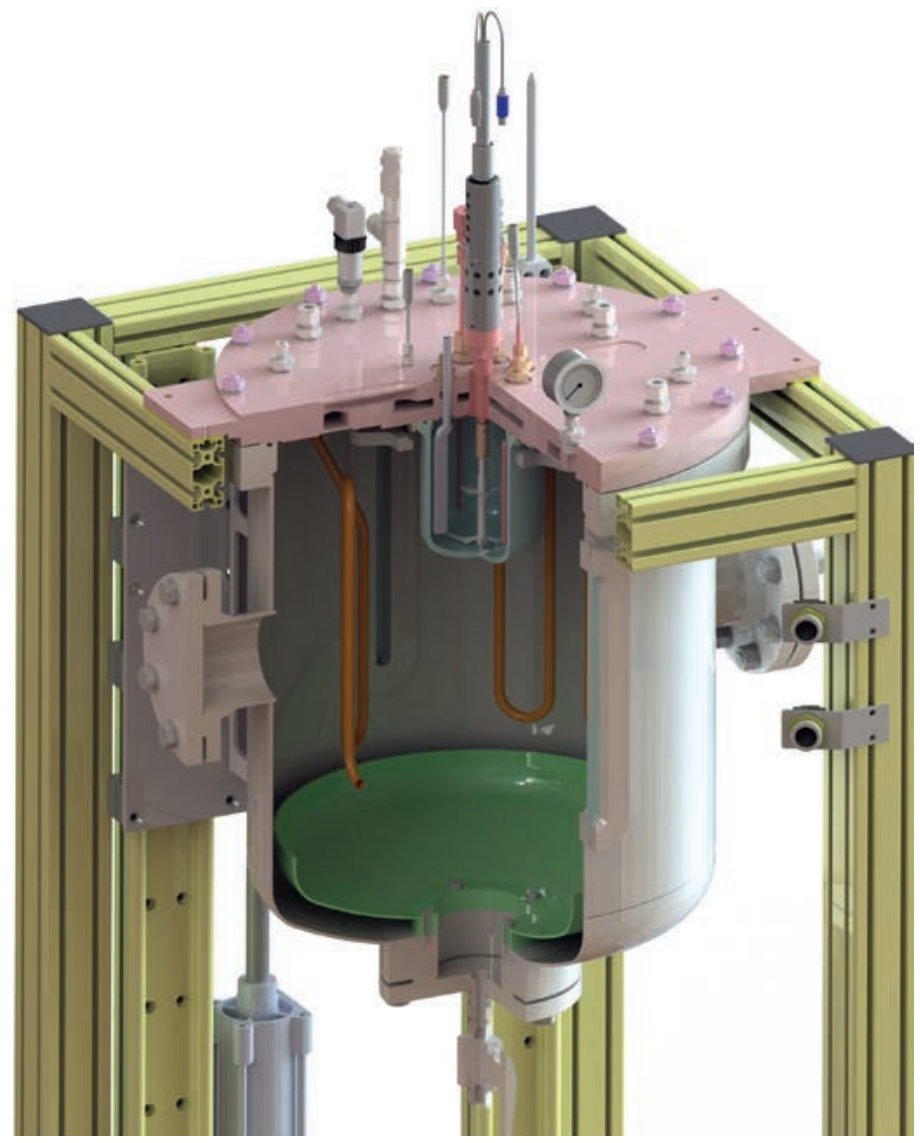

Fig. 7. CAD drawing of final scale-down reactor design (Premex Solutions $\mathrm{GmbH}$ ). The geometrically similar glass vessel (slightly blue) is visualized inside the safety container. The $\mathrm{H} / \mathrm{C}$-finger and the agitator are also shown.

of the process and to establish the design space. Some or all of those PAR experiments may be replaced by simulations and a few scale-down experiments to validate the simulations. With this systematic approach to development (QbD) the normal operating range (NOR), the PAR and also the design space for the reaction process parameters can be defined. ${ }^{[8,9]}$
Fig. 8. Schematic representation of scale-down reactor assembly. B1.P1 and B1.T1 are allocated to the inside of the safety container B1. The dip tube and R1.T1 are allocated to the inside of the glass vessel $\mathrm{R} 1$.

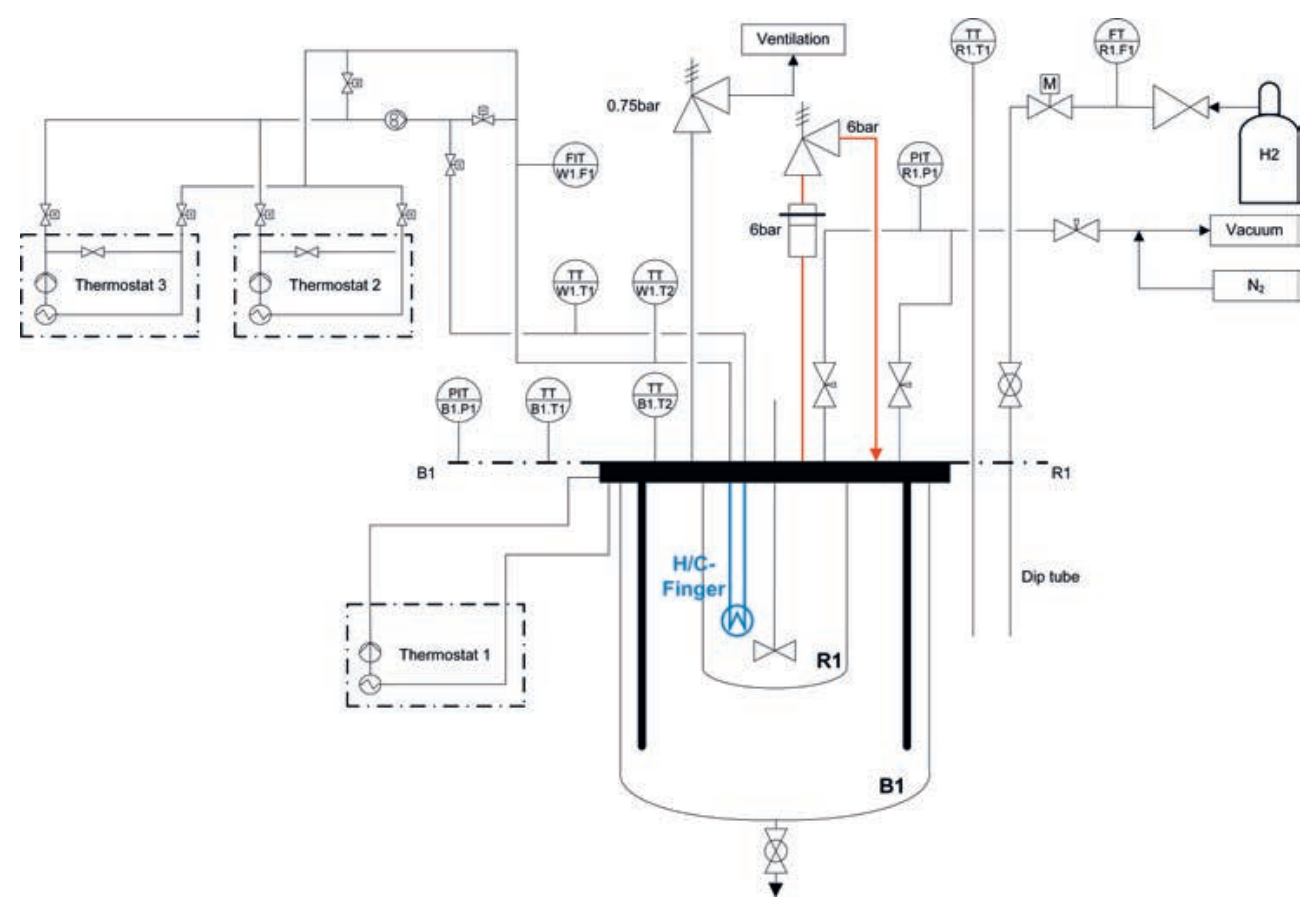




\subsection{Direct Scale-up to Production-Scale}

If the results of the simulation and the scale-down experiments agree, the reaction process can be scaled-up directly to production scale. In comparison to the classical scale-up strategy only a few laboratory-scale and no pilot-scale experiments are required for the whole process scale-up. By reducing the number of experiments, especially on a pilot-scale, indirect costs such as the cost of environmental pollution are considerably reduced in addition to costs for operation for well-known reasons. Furthermore, the time to market is reduced considerably.

\subsection{Production-Scale Data}

The data generated from the production-scale reaction processes can be used to continuously validate and calibrate the dynamic process model. For this, the measured concentrations and temperature profiles can be compared with the results from the simulation and the scale-down experiment. If a significant deviation is observed, the optimized parameters of the process model can be adjusted accordingly.

\subsection{Process Monitoring}

The data observed during the production-scale process can be compared with the simulated prediction. If temperature and/ or concentration profiles deviate strongly from the simulation, potential problems will be detected. With such early detection it is possible to intervene in the process and save the batch. The model makes the chemical reaction predictable on a large scale, so deviations give hints that the reaction performance is influenced by factors which were not evaluated during laboratory-scale development. This may be catalyst poisoning from a starting material, or different catalyst turnover for example. In the classical approach, these effects will only be detected when a huge number of reactions is analysed statistically. The scale-down strategy on the other hand enables a systematic and efficient investigation of the root cause.

\section{Conclusion and Outlook}

With the proposed novel scale-up strategy for hydrogenation reactions in agitated multipurpose autoclaves it will be possible to efficiently scale-up reaction processes from laboratory to production-scale. The combination of advanced scale-down lab experiments and a powerful computational model for the reaction and scale-up process enables Siegfried to make scale-up processes more reliable and predictable with even fewer experiments. Predictions of the chemical process model are used to optimize process parameters and evaluate robust operating ranges for the hydrogenation processes in production-scale. Thanks to the collaboration with the FHNW it was already possible for Siegfried to represent reactions which could not be simulated with commercially available software alone. With advanced scale-down experiments in a custom-designed scale-down reactor it will also be possible to validate those simulations in laboratory-scale.

This article presents a general overview of the proposed scaleup strategy. Furthermore, the heat transfer part of the improved process model was described in detail. Likewise, the design of the customized scale-down reactor including the $\mathrm{H} / \mathrm{C}$-finger was discussed.

All parts of the project as yet not completed will be described in a future publication. This includes a further investigation of the dynamic process model's mass transfer part. Mass transfer will be incorporated into the model in the same way as the heat transfer part and hence the dependency of the physical properties of the reaction mixture on the gas-liquid mass transfer will be considered. Furthermore, the constructed scale-down reactor and the validation of the scale-up strategy by proof of concept with model reactions will be described.

\begin{tabular}{ll} 
Symbols and & Subscripts \\
Symbol & Description \\
$A$ & Heat transfer area \\
$c$ & Concentration \\
$C_{1}-C_{5}$ & Parameters for Nusselt numbers \\
$c p$ & Specific heat capacity \\
$d_{A}$ & Agitation diameter \\
$d_{c}$ & Mean diameter of jacket coil helix \\
$d_{e}$ & Annular space width of jacket \\
$d_{V}$ & Inner vessel diameter \\
$k_{L} a$ & Overall mass transfer coefficient \\
$m$ & Mass \\
$\dot{m}$ & Mass flow \\
$n$ & Agitation speed \\
$N u$ & Nusselt number \\
$P r$ & Prandtl number, Pr $=\frac{\eta * c p}{\lambda}$ \\
$\dot{Q}$ & Heat flow \\
$R e$ & Reynolds number \\
$t$ & Time \\
$T$ & Absolute temperature \\
$U$ & Heat transfer coefficient \\
$v$ & Velocity \\
$\eta$ & Dynamic viscosity \\
$\lambda$ & Thermal conductivity \\
$\rho$ & Density \\
Subscript & \\
$1 / 2$ & 1 or 2 \\
$a c c u$ & Due to accumulation \\
$i n l e t$ & Entering heat transfer fluid \\
$J$ & Jacket / Heat transfer fluid \\
$J, W$ & Heat transfer fluid near reactor wall \\
$L$ & Liquid side \\
$R$ & Reaction mixture \\
$r e a c t$ & Due to reactions \\
$R W$ & From reaction mixture to reactor wall \\
$R, W$ & Reaction mixture near reactor wall \\
sat & Saturation \\
$W$ & Reactor wall \\
$W J$ & From reactor wall to heat transfer fluid \\
& \\
& \\
\hline &
\end{tabular}

\section{Background and Acknowledgements}

The authors thank the Aargau Research Fund for their financial support of this project, which is a joint effort between academia (FHNW) and industry (Siegfried AG). We would like to thank all those involved from both partners for their assistance and support.

Furthermore, we would like to thank Premex Solutions $\mathrm{GmbH}$, Andreas Stubbe (HS Labortechnik $\mathrm{GmbH}$ ) and the workshop of the FHNW Muttenz for their huge support with the design and construction of the scale-down reactor shown in Fig. 7 and Fig. 8. We would like to thank the Institute of Thermal and Fluid Engineering of the FHNW for the support with the CFD calculations for the design of the H/C-finger (Fig. 6). Also, we would like to thank Ronny Hardegger and Andrew Brown for their constructive criticism of the manuscript.

The scale-down reactor presented within this article is a joint development of several companies working together under the lead of the FHNW HLS (School of Life Sciences). The members share their ideas in order to develop new technologies within the field of process development. To manage intellectual property and to make new technologies available to the process industry, the association Miniplant 4.0 was founded in 2019. This association enables the transfer of new technologies and processes developed at the FHNW HLS to practice in close collaboration with local companies. The association Miniplant 4.0 thus strengthens the role of the FHNW HLS in the field of translational research. If you are interested in the association Miniplant 4.0, do not hesitate to contact Prof. Dr. Andreas Zogg directly.

This article is dedicated to our valued colleague Dr. Beat Weber. He was a great chemist who contributed significantly to the improvement of process development. ${ }^{[28]}$ Furthermore, he was a key person in the long history of the collaboration between Siegfried and FHNW. 
The development of the scale-down reactor shown in Fig. 7 and Fig. 8 was initiated in the 'New Technology' Group at F. Hoffmann-La Roche AG in 2012. The first prototype reactor was designed to investigate the adiabatic behaviour of a reaction mass after a chlorination step. The device was further developed with several apprentices and students in the fields of measuring and control technology, process engineering and chemical engineering, as well as laboratory technicians. In 2018 F. Hoffmann-La Roche AG transferred the reactor set-up to the FHNW HLS, keeping a privileged right to use. This donation opened the door for further development of the scale-down reactor at the FHNW HLS and thus made the present project possible. Therefore, the authors would like to thank all the people from F. Hoffmann-La Roche AG involved in the development as well as the handover of the scale-down-reactor to the FHNW HLS. Especially we would like to thank Christian Walch, Cédric Hutter, Andreas Riess, Ivo \& Karl-Heinz Langendorf, Jérome Blum, Eik Prenzlow, Lukas Kündig, Christoph Loy, Florian Nestler, Andreas Heilmann, Tobias Kratz, Micha Bischofberger, Rainer Nicolay, Stefan Hildbrandt, and Michelangelo Scalone. Especially we would also like to thank Prof. Dr. David Zogg for his support during many student projects carried out at the FHNW-HT (School of Engineering).

Fig. 6 (right) used with courtesy of ANSYS, Inc.

Received: August 31, 2021

[1] J.-P. Euzen, P. Trambouze, J.-P. Wauquier, 'Scale-up methodology for chemical processes', Editions Technip, Paris, 1993.

[2] M. Levin, 'Pharmaceutical process scale-up', Marcel Dekker Inc., New York, 2002.

[3] D. J. am Ende, C. B. Seymour, T. J. N. Watson, J. Pharm. Innov. 2010, 5, 72, https://doi.org/10.1007/s12247-010-9083-1.

[4] J. R. Bourne, Org. Process Res. Dev. 2003, 7, 471, https://doi.org/10.1021/op020074q.

[5] C. W. Mitchell, J. D. Strawser, A. Gottlieb, M. H. Millonig, F. A. Hicks, C. D. Papageorgiou, Org. Process Res. Dev. 2014, 18, 1828, https://doi.org/10.1021/op500207r.

[6] R. N. Landau, D. G. Blackmond, H.-H. Tung, Ind. Eng. Chem. Res. 1994, 33, 814, https://doi.org/10.1021/ie00028a005.

[7] Accelerated Scale-up, Reaction calorimetry and reactor simulation, https://www.mt.com/mt_ext_files/Editorial/Generic/2/wr_publication_51724866_865_Bollyn_Editorial-Generic_1143193241121_ files/51724866.pdf, accessed July 3, 2021.

[8] S. Tummala, A. Ramirez, S. Srivastava, D. M. Hallow, in 'Chemical Engineering in the Pharmaceutical Industry', Eds. D. J. am Ende, M. T. am Ende, John Wiley \& Sons, Inc., Hoboken, NJ, USA, 2019, pp. 1091, https://doi.org/ 10.1002/9781119600800.ch50.

[9] J. L. Burt, A. D. Braem, A. Ramirez, B. Mudryk, L. Rossano, S. Tummala, J. Pharm. Innov. 2011, 6, 181, https://doi.org/10.1007/s12247-011-9109-3.

[10] ICH guideline Q8 (R2) on pharmaceutical development, https://www.ema. europa.eu/en/documents/scientific-guideline/international-conference-harmonisation-technical-requirements-registration-pharmaceuticals-human-use_en-11.pdf, accessed August 4, 2021.
[11] A Glimpse into Real-Time Methanol Synthesis: Dynamic Operation of a Miniplant at Fraunhofer ISE, https://www.ise.fraunhofer.de/content/dam/ ise/en/documents/press-releases/2020/0920_ISE_PR_Methanolplant.pdf, accessed August 10, 2021

[12] 'Handbuch der Rührtechnik: Grundlagen, Auslegung, Rührer und Rührsysteme, Mechanik, Konstruktion, Dichtungstechnik, Betriebssicherheit, Anwendungsgebiete', Ed. P. Hentrich, EKATO RührUnd Mischtechnik GmbH, Schopfheim, 2000.

[13] M. Zlokarnik, in 'Ullmann's Encyclopedia of Industrial Chemistry', Ed. Wiley-VCH Verlag GmbH \& Co. KGaA, Wiley-VCH Verlag $\mathrm{GmbH} \&$ Co. KGaA, Weinheim, Germany, 2003, pp. b02-25, https://doi.org/10.1002/14356007.b02_25.

[14] B. Zufferey, PhD Thesis Swiss Federal Institute of Technology Lausanne (EPFL) No. 3464, 2006.

[15] T. Kupr, L. Hub, Angew. Chem. Thermodyn. Thermoanal. 1979, 37, 334, https://doi.org/10.1007/978-3-0348-5545-7.

[16] The Constant Flux Reactor, http://www.iptonline.com/articles/public/ AsheMorrisLtd.pdf, accessed July 7, 2021.

[17] W. Regenass, CHIMIA 1997, 51, 189.

[18] A. Zogg, F. Stoessel, U. Fischer, K. Hungerbühler, Thermochim. Acta 2004, 419, 1, https://doi.org/10.1016/j.tca.2004.01.015.

[19] S. Holz, L. Stewers, H. Thielert, Z. Guetta, J. Repke, Chem. Ing. Tech. 2020, 92, 209, https://doi.org/10.1002/cite.201900063.

[20] Dynochem Version 6.0, Scale-Up Systems Ltd., Dublin, Ireland, 2021.

[21] MATLAB Version 9.9.0.1570001 (R2020b), The MathWorks Inc., Natick, Massachusetts, 2021.

[22] Verein Deutscher Ingenieure, 'VDI heat atlas', Springer, Berlin Heidelberg, 2010.

[23] R. F. Dream, Chem. Eng. 1999, 106, 90.

[24] Surrogate Optimization Algorithm, https://ch.mathworks.com/help/gads/ surrogate-optimization-algorithm.html, accessed June 21, 2021.

[25] E. L. Cussler, 'Diffusion: mass transfer in fluid systems', Cambridge University Press, New York, 2009.

[26] ANSYS® Fluent ${ }^{\circledR}$ Version R19.2, ANSYS Inc., Southpointe, Pennsylvania, 2018.

[27] G. T. Benz, Chem. Eng. Prog. 2008, 104, 32

[28] C. Adler, J. Brunner, C. Fichtner, P. Küng, M. K. Levis, H.-R. Ruchti, A. Sjöberg, B. Weber, Chim. Int. J. Chem. 2006, 60, 523, https://doi.org/10.2533/chimia.2006.523.

\section{License and Terms}

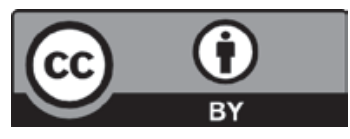

This is an Open Access article under the terms of the Creative Commons Attribution License CC BY 4.0. The material may not be used for commercial purposes.

The license is subject to the CHIMIA terms and conditions: (http:// chimia.ch/component/sppagebuilder/?view=page \&id=12).

The definitive version of this article is the electronic one that can be found at https://doi.org/10.2533/chimia.2021.948 